\title{
Multi-Core SVM Fault Diagnosis Of Electrical System Based on Dimension Directed Factor Graph
}

\author{
Liu Hongjun, Guo Jinran \\ Department of International Education Institute, North China Electric Power University, Beijing, \\ 102206, China
}

Keywords: electrified railway; traction transformer; load characteristic; simulation

\begin{abstract}
According to the unbalanced load of traction power supply circuit of electrified railway, this paper takes the impedance matching balance traction transformer of high-speed passenger rail lines as the research object. Based on the voltage balance equation, winding connection principle and symmetrical component method of transformer, the load current unbalance characteristic of the balanced traction transformer is studied; according to the specific working conditions of high-speed passenger rail lines, the variation characteristics of the load characteristics of the balanced traction transformer are analyzed, providing a reference for the operation and control of high-speed passenger rail lines
\end{abstract}

\section{Introduction}

The load of electric traction locomotive belongs to single-phase rectification type load. Two phases in a three-phase system are used in a single locomotive. One phase is used as a traction network, and the other is connected to the earth as a ground rail loop. Such work method will inevitably lead to negative sequence current due to unbalanced three-phase load, while the unbalanced operation of the power supply system of railway traction network will affect the safety, stability and economic operation of the railway power supply system and electric system. Meanwhile, the load of traction transformer is fluctuated and random, the average load rate of transformer in traction substation is low, but the short-time overload rate is higher, and the traction load current contains higher harmonic component. Therefore, it is urgent to study the variation law of load characteristics of balanced traction transformer and formulate corresponding measures, in order to reduce the influence degree of power system and ensure the reliable power supply for power users.

\section{Voltage transformer wiring principle and load current unbalance degree analysis}

The basic wiring principle of impedance matching balance traction transformer is shown in Figure $1 . W_{1}$ and $W_{2}$ are the numbers of turns of winding for primary side and secondary side of traction transformer, and $W_{3}$ is the number of turns of winding of exterior traction side, and is winded on the ab winding iron core column. Triangle connection structure of secondary side, is to add two outer windings to the non earthing phase, and the two ends are connected to the two-phase bus of secondary side, and it provides power of network to two sides of power supply arm. 


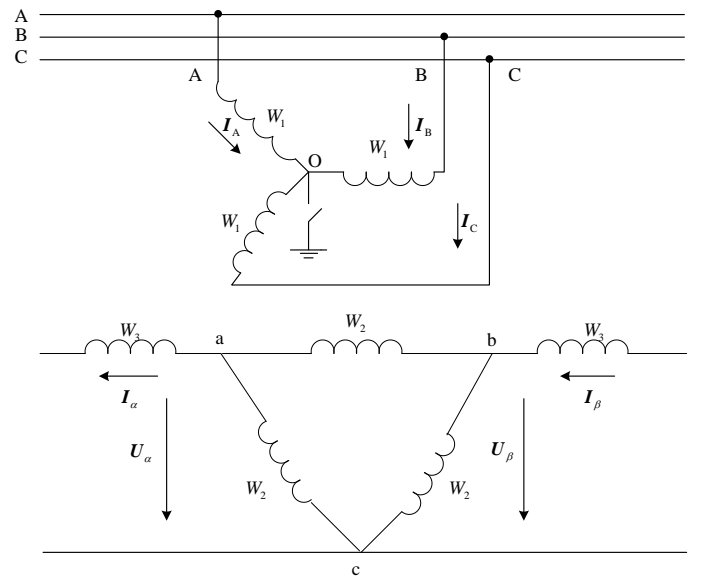

Fig.1 Connection principle diagram of impedance-matched balance traction transformers

According to the wiring principle of Figure 1, the relationship between the primary and secondary sides of the transformer can be seen as follows

$$
\left\{\begin{array}{l}
\boldsymbol{I}_{A} W_{1}-\boldsymbol{I}_{a} W_{2}=0 \\
\boldsymbol{I}_{B} W_{1}-\boldsymbol{I}_{b} W_{2}=-\left(\boldsymbol{I}_{\alpha}+\boldsymbol{I}_{\beta}\right) \\
\boldsymbol{I}_{C} W_{1}-\boldsymbol{I}_{c} W_{2}=0
\end{array}\right.
$$

In which $W_{3}=\frac{1}{2}(\sqrt{3}-1) W_{2}, \quad \boldsymbol{I}_{\mathrm{A}}, \quad \boldsymbol{I}_{\mathrm{B}}$ and $\quad \boldsymbol{I}_{\mathrm{C}}$ are three-phase alternating currents on the primary side for impedance-matched balance traction transformers; $\boldsymbol{I}_{\alpha}$ and $\boldsymbol{I}_{\beta}$ are the currents of $\alpha$ phase and $\beta$ phase on the secondary side, $\boldsymbol{U}_{\alpha}$ and $\boldsymbol{U}_{\beta}$ are the voltages of $\alpha$ phase and $\beta$ phase on the secondary phase.

From the impedance balance traction transformer voltage balance equation and winding connection principle, the transformation relation on the phase current of the transformer between the primary side and the secondary side can be obtained as

$$
\boldsymbol{I}_{A B C}=\boldsymbol{Q} \boldsymbol{I}_{\alpha \beta}
$$

In which ${ }^{\boldsymbol{I}_{A B C}}$ is the phase current matrix of primary side of traction transformer; ${ }^{\boldsymbol{I}_{\beta}}$ is the phase current matrix of secondary side of traction transformer; $\boldsymbol{Q}$ is the transformation matrix of phase currents of traction transformer on the primary and secondary sides, and the transformation matrix of traction transformer phase current $Q$ is:

$$
Q=\frac{1}{2 \sqrt{3} K}\left[\begin{array}{ll}
\sqrt{3}+1 & 1-\sqrt{3} \\
-2 & -2 \\
1-\sqrt{3} & \sqrt{3}+1
\end{array}\right]
$$

In which $K$ is the ratio of primary and secondary side windings of traction transformer. According to the symmetrical component method, the relation between three-phase current and positive sequence current, negative sequence current and zero sequence current can be obtained as:

$$
\boldsymbol{I}_{120}=\boldsymbol{P} \boldsymbol{I}_{A B C}
$$

In which symmetric component matrix $\boldsymbol{P}=\frac{1}{3}\left[\begin{array}{ccc}1 & \alpha & \alpha^{2} \\ 1 & \alpha^{2} & \alpha \\ 1 & 1 & 1\end{array}\right]$, operator $\alpha=e^{j 120^{\circ}}, \quad \alpha^{2}=e^{j 240^{\circ}}$, and $1+\alpha+\alpha^{2}=0$. From the formula (4) and the formula (5), the relationship between positive sequence current, negative sequence current, zero sequence current and impedance matching balance traction transformer two side phase current is as follows:

$$
\boldsymbol{I}_{120}=\boldsymbol{M I}_{\alpha \beta}
$$


In which $\boldsymbol{M}$ is transformation matrix of side current of secondary side of positive sequence current, negative sequence current, zero sequence current and impedance matching traction transformer, and the transformation matrix is $\boldsymbol{M}$ :

$$
\boldsymbol{M}=\frac{1}{6 \sqrt{3} K}\left[\begin{array}{cc}
1-\sqrt{3}) \alpha^{2}-2 \alpha+\sqrt{3}+1 & (1+\sqrt{3}) \alpha^{2}-2 \alpha+1-\sqrt{3} \\
-2 \alpha^{2}+(1-\sqrt{3}) \alpha+\sqrt{3}+1 & -2 \alpha^{2}+(1+\sqrt{3}) \alpha+1-\sqrt{3} \\
0 & 0
\end{array}\right]
$$

If $I^{+}$is effective value of positive sequence current, $I^{-}$is effective value of negative sequence current, $\alpha_{z} 、 \beta_{z}$ are impedance angles of $\alpha$ phase and ${ }^{\beta}$ phase of secondary side, according to (4), the current unbalance degree of traction transformer with impedance matching balance can be deduced as:

$$
\varepsilon=\frac{I^{-}}{I^{+}}=\sqrt{\frac{I_{\alpha}{ }^{2}+I_{\beta}{ }^{2}-2 I_{\alpha} I_{\beta} \cos \left(\alpha_{Z}-\beta_{Z}\right)}{I_{\alpha}{ }^{2}+I_{\beta}{ }^{2}+2 I_{\alpha} I_{\beta} \cos \left(\alpha_{Z}-\beta_{Z}\right)}}
$$

According to the formula (5), the load current unbalance degree of the impedance matching balance traction transformer is related to the load size and the load power factor angle.

\section{Load current unbalance characteristics of high speed passenger train}

In order to further analyze the relationship between the current imbalance and the traction current in the left and right traction power supply arm of the secondary side, during the operation of the high speed passenger dedicated line, this paper combines and discusses three cases of high speed passenger train operation, and the definition of current ratio of $\alpha$ phase and ${ }^{\beta}$ phase are:

$$
D=\frac{I_{\alpha}}{I_{\beta}}
$$

(1) When the impedance angle of two phase load of secondary side is equal and the load current amplitude is unequal:

$$
\left\{\begin{array}{l}
\alpha_{z}=\beta_{z} \\
I_{\alpha} \neq I_{\beta}
\end{array}\right.
$$

For the general case, take $I_{\alpha}=D I_{\beta}$ and $0 \leq D \leq 1$, and the current unbalance degree can be obtained by substituting the formula (7) into (5):

$$
\varepsilon=\frac{I^{-}}{I^{+}}=\frac{\left|I_{\alpha}-I_{\beta}\right|}{I_{\alpha}+I_{\beta}}=\frac{D-1}{D+1}
$$

According to the formula (8), the change curve of current unbalance with current ratio $\mathrm{D}$ can be drawn as shown in figure 2:

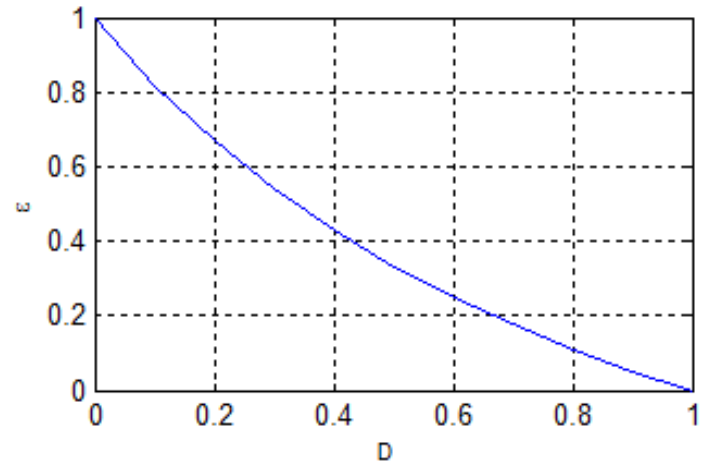

Fig.2 Change curve diagram of $\alpha_{z}=\beta_{z}, I_{\alpha} \neq I_{\beta}$

(2) When the impedance angle of two-phase load on the secondary side is not equal and the load current amplitude is equal: 


$$
\left\{\begin{array}{l}
\alpha_{{ }_{\mathrm{z}}} \neq \beta_{\mathrm{z}} \\
I_{\alpha}=I_{\beta}
\end{array}\right.
$$

Make the load impedance angle of $\alpha$ side varies between $-90^{\circ} \sim+90^{\circ}$, take the fixed value to the load impedance angle $\beta_{z}$ on the $\beta$ side, and substitute the formula (9) into (5).

When $\beta_{\mathrm{z}}=-90^{\circ}$ the expression of the current unbalance degree is:

$$
\varepsilon_{1}=\frac{\sqrt{1-\cos \left(\alpha_{z}+90^{\circ}\right)}}{\sqrt{1+\cos \left(\alpha_{z}+90^{\circ}\right)}}
$$

When $\beta_{z}=0^{\circ}$ the expression of the current unbalance degree is:

When $\beta_{\mathrm{z}}=90^{\circ}$ the expression of the current unbalance degree is:

$$
\varepsilon_{2}=\frac{\sqrt{1-\cos \alpha_{z}}}{\sqrt{1+\cos \alpha_{z}}}
$$

$$
\varepsilon_{3}=\frac{\sqrt{1-\cos \left(\alpha_{z}-90^{\circ}\right)}}{\sqrt{1+\cos \left(\alpha_{z}-90^{\circ}\right)}}
$$

According to the formula (10) (11) and (12), the change curve of current unbalance with current ratio $\mathrm{D}$ is shown in figure 3 :

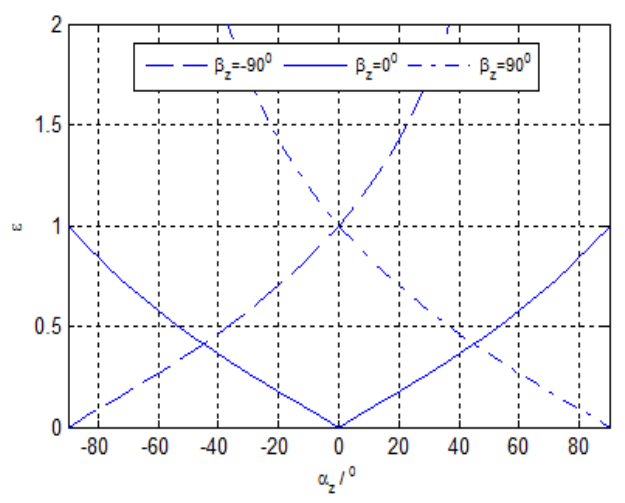

Fig.3 Change curve diagram of $\alpha_{{ }_{2}} \neq \beta_{z}, I_{\alpha}=I_{\beta}$

(3) When the impedance angle of two phase load on the secondary side is unequal, and the load current amplitude is not equal:

$$
\left\{\begin{array}{l}
\alpha_{z} \neq \beta_{z} \\
I_{\alpha} \neq I_{\beta}
\end{array}\right.
$$

Make the load impedance angle of $\alpha$ side varies between $-90^{\circ} \sim+90^{\circ}$, take the load impedance angle $\beta_{z}=-45^{\circ}$ on the $\beta$ side, and substitute the formula (13) into (5).

When $\mathrm{D}=0$, the current unbalance degree is expressed as follows:

$$
\varepsilon_{1}=1
$$

When $\mathrm{D}=0.5$ the current unbalance degree is expressed as follows:

$$
\varepsilon_{2}=\frac{\sqrt{1.25-\cos \left(\alpha_{z}+45^{\circ}\right)}}{\sqrt{1.25+\cos \left(\alpha_{z}+45^{\circ}\right)}}
$$

When $\mathrm{D}=1$ the current unbalance degree is expressed as follows:

$$
\varepsilon_{3}=\frac{\sqrt{2-2 \cos \left(\alpha_{z}+45^{\circ}\right)}}{\sqrt{2+2 \cos \left(\alpha_{z}+45^{\circ}\right)}}
$$


According to the formula (14) (15) and (16), the change curve of current unbalance with current ratio $\mathrm{D}$ is shown in figure 4 :

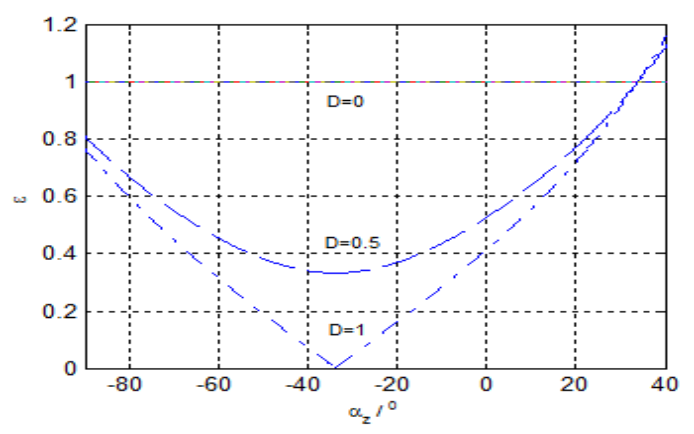

Fig.4 Change curve diagram of $\alpha_{z} \neq \beta_{z}, I_{\alpha} \neq I_{\beta}$

It can be seen from the figure 2, 3 and 4 that, the nearer the traction current amplitude of the secondary-side left and right traction power supply arm is, the closer the load impedance angle of both sides is, the smaller the current imbalance degree is. When power factor angle of two arms load is near the area of $\alpha_{z}-\beta_{z}=0^{\circ}$, the minimum degree of current imbalance is obtained. When the operation of the train is near the area of $\alpha_{z}-\beta_{z}= \pm 90^{\circ}$, namely, the load power factor angle difference of two traction power supply arm is $90^{\circ}$, the maximum current unbalance of the impedance matching traction transformer is 1 , the negative sequence current generated by the impedance matching balance traction transformer is basically equal to the positive sequence current with the operation of traction, and the negative sequence current reaches the maximum value. Under this condition, the traction power supply network has the greatest impact, leading to the occurrence of railway accidents easily.

\section{Simulation experiment of load current unbalance degree}

An experimental model of impedance matching balanced traction transformer shown in Figure 1 is built on Matlab/Simulink platform. By using this experimental model, this paper analyzes the different power consumption of electrified railway under different operating conditions, and current imbalance of impedance matching balance traction transformer. Take the load is variable power, and take it as pure active power, and the reactive power is close to zero, then the experimental results are shown in table 1.The relation between the power change of the two side load and the unbalance degree of the current can be plotted by Table 1, as shown in figure 6 .

Table1 Experimental data of current unbalanced degree of impedance-matched balance traction transformers

\begin{tabular}{|c|c|c|c|c|c|}
\hline \multicolumn{3}{|c|}{$(\mathrm{MW})$} & \multicolumn{3}{c|}{ (A) } \\
\hline$P_{\alpha}$ & $P_{\beta}$ & $P_{\alpha} / P_{\beta}$ & $I^{+}$ & $I^{-}$ & $\varepsilon$ \\
\hline 15.125 & 15.125 & 1 & 156.2 & 0.119 & 0.001 \\
& & & & & \\
\hline 18.906 & 15.125 & 1.25 & 174.8 & 18.72 & 0.107 \\
\hline 25.208 & 15.125 & 1.67 & 204.4 & 49.31 & 0.241 \\
\hline 37.813 & 15.125 & 2.50 & 257.7 & 106.1 & 0.412 \\
\hline 75.625 & 15.125 & 5 & 368.1 & 233.4 & 0.634 \\
& & & & & \\
\hline 0 & 15.125 & 0 & 78.37 & 77.78 & 0.992 \\
\hline
\end{tabular}




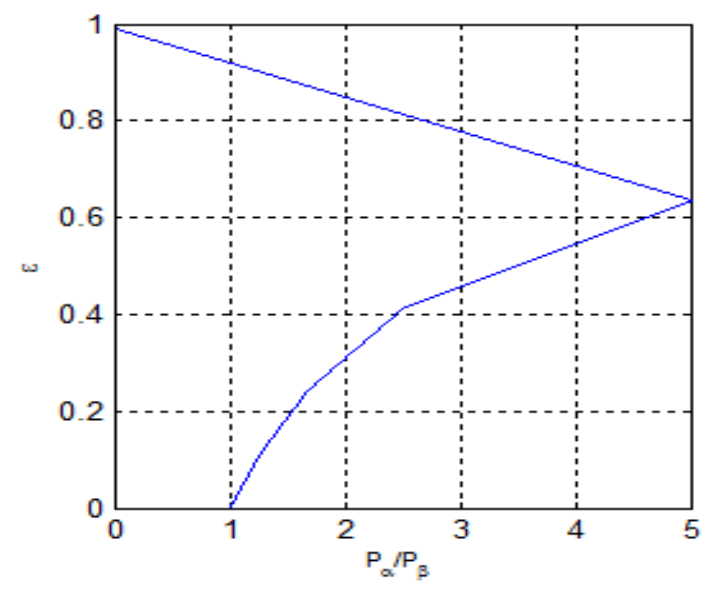

Fig.6 The relationship of Load power ratio and the current unbalanced degree

From Table 1 and Figure 6, the relation between the load power ratio and the current unbalance of the traction transformer is obtained by impedance matching, with the distribution of passenger flow in different time periods and different regions, and the operation of different marshalling modes of trains, the current unbalance degree of the impedance matching balance traction transformer also changes. When the power supply arm is empty, the current unbalance reaches the maximum value of 1 ; when two phase load of secondary phase is the same, namely, the load power ratio is 1 , and the current unbalance reaches the minimum value of 0 .

\section{Conclusion}

(1) The negative sequence current of the power system caused by the traction transformer with impedance matching is related to the load size, phase difference and other factors of traction of the two arms.

(2) In the case of no load for any traction arm, the current imbalance of the impedance matching balance traction transformer is nearly 1 ; when the load phase of the two phase power supply arm is unbalanced, the current imbalance reaches 0 . At this time, in traction operation, the impedance matching balance traction transformer produces less negative sequence current and less harmonic current, and causes the least influence on traction power supply network.

(3) When the high speed passenger dedicated line runs at $D=0$, the negative sequence current generated by the impedance matching balance traction transformer is equal to the positive sequence current, and the negative sequence current reaches the maximum value, which has the greatest impact on the traction power supply network, leading to the occurrence of the railway accident.

\section{References}

[1] Weisen Pan, Shizhan Chen, Zhiyong Feng. Investigating the Collaborative Intention and Semantic Structure among Co-occurring Tags using Graph Theory. 2012 International Enterprise Distributed Object Computing Conference, IEEE, Beijing, pp. 190-195.

[2] Jennifer W. Chan, Yingyue Zhang, and Kathryn E. Uhrich, Amphiphilic Macromolecule Self-Assembled Monolayers Suppress Smooth Muscle Cell Proliferation, Bioconjugate Chemistry, 2015, 26(7), 1359-1369.

[3] Yingyue Zhang, Evan Mintzer, and Kathryn E. Uhrich, Synthesis and Characterization of PEGylated Bolaamphiphiles with Enhanced Retention in Liposomes, Journal of Colloid and Interface Science, 2016, 482, 19-26.

[4] Jonathan J. Faig, Alysha Moretti, Laurie B. Joseph, Yingyue Zhang, Mary Joy Nova, Kervin Smith, and Kathryn E. Uhrich, Biodegradable Kojic Acid-Based Polymers: Controlled Delivery of Bioactives for Melanogenesis Inhibition, Biomacromolecules, 2017, 18(2), 363-373. 